TRANSACTIONS OF THE

AMERICAN MATHEMATICAL SOCIETY

Volume 350, Number 4, April 1998, Pages 1379-1393

S 0002-9947(98)02094-7

\title{
GEOMETRIC FAMILIES OF CONSTANT REDUCTIONS AND THE SKOLEM PROPERTY
}

\author{
BARRY GREEN
}

\begin{abstract}
Let $F \mid K$ be a function field in one variable and $\mathcal{V}$ be a family of independent valuations of the constant field $K$. Given $v \in \mathcal{V}$, a valuation prolongation $\mathrm{v}$ to $F$ is called a constant reduction if the residue fields $F \mathrm{v} \mid K v$ again form a function field of one variable. Suppose $t \in F$ is a non-constant function, and for each $v \in \mathcal{V}$ let $V_{t}$ be the set of all prolongations of the Gauß valuation $v_{t}$ on $K(t)$ to $F$. The union of the sets $V_{t}$ over all $v \in \mathcal{V}$ is denoted by $\boldsymbol{V}_{t}$.

The aim of this paper is to study families of constant reductions $\boldsymbol{V}$ of $F$ prolonging the valuations of $\mathcal{V}$ and the criterion for them to be principal, that is to be sets of the type $\boldsymbol{V}_{t}$. The main result we prove is that if either $\mathcal{V}$ is finite and each $v \in \mathcal{V}$ has rational rank one and residue field algebraic over a finite field, or if $\mathcal{V}$ is any set of non-archimedean valuations of a global field $K$ satisfying the strong approximation property, then each geometric family of constant reductions $\boldsymbol{V}$ prolonging $\mathcal{V}$ is principal. We also relate this result to the Skolem property for the existence of $\mathcal{V}$-integral points on varieties over $K$, and Rumely's existence theorem. As an application we give a birational characterization of arithmetic surfaces $\mathcal{X} / S$ in terms of the generic points of the closed fibre. The characterization we give implies the existence of finite morphisms to $\mathbb{P}_{S}^{1}$.
\end{abstract}

\section{INTRODUCTION}

Let $F \mid K$ be a function field in one variable and $\mathcal{V}$ be a family of independent valuations of the exact constant field $K$. Given $v \in \mathcal{V}$, a valuation prolongation $\mathrm{v}$ to $F$ is called a constant reduction of $F$ if the residue fields $F \mathrm{v} \mid K v$ again form a function field of one variable. Suppose $t \in F$ is a non-constant function and for each $v \in \mathcal{V}$ let $V_{t}$ be the set of all prolongations of the Gauß valuation $v_{t}$ on $K(t)$ to $F$. We denote the union of the sets $V_{t}$ over all $v \in \mathcal{V}$ by $\boldsymbol{V}_{t}$.

Suppose $\boldsymbol{V}$ is a family of constant reductions of $F$ prolonging the valuations of $\mathcal{V}$. Then $\boldsymbol{V}$ is said to be geometric if there is a non-constant function $t \in F$ such that $\boldsymbol{V}$ and $\boldsymbol{V}_{t}$ are almost equal, i.e., the symmetric difference of $\boldsymbol{V}$ and $\boldsymbol{V}_{t}$ is finite. The family $\boldsymbol{V}$ is said to be principal if $\boldsymbol{V}=\boldsymbol{V}_{t}$ for some non-constant function $t \in F$.

Our aim in this paper is to prove the following theorem:

Received by the editors December 5, 1995.

1991 Mathematics Subject Classification. 11G30, 11R58, 12J10, 14G25.

This paper is part of the author's Habilitation Thesis, University of Heidelberg, January 1995. The author would like to thank the Deutsche Forschungsgemeinschaft and the University of Heidelberg for supporting this work. 
Theorem 1. Let $F \mid K$ be a function field in one variable, $\mathcal{V}$ a family of independent valuations of $K$ and $\boldsymbol{V}$ a geometric family of constant reductions of $F$ prolonging $\mathcal{V}$. Suppose that one of the following conditions is satisfied:

i) $\mathcal{V}$ consists of only one valuation with respect to which $K$ is henselian.

ii) $\mathcal{V}$ is finite and $K$ is algebraically closed.

iii) $\mathcal{V}$ is finite and each valuation $v \in \mathcal{V}$ has rational rank one and residue field algebraic over a finite field.

iv) $\mathcal{V}$ is a set of non-archimedean valuations of a global field $K$ satisfying the strong approximation property. Thus for number fields we can take $\mathcal{V}$ to be the set of all non-archimedean valuations and for function fields the set of all but one.

Then the family $\boldsymbol{V}$ is principal.

The approach we follow in proving this result corresponds to the axiomatic approach from $[\mathrm{G}-\mathrm{P}-\mathrm{R}]$ to Rumely's Local Global Principle. Hence the proof is divided into two parts, the first containing a proof of the result (actually a stronger form) in the semi-local situation, i.e., when $\mathcal{V}$ is finite, and the second the passage from the semi-local to the global situation.

In the local context, when $\mathcal{V}$ consists of only one valuation, the assertion that $\boldsymbol{V}$ is principal is equivalent to the solvability of local Skolem problems. This relationship, together with the proof of the theorem for this case, has been studied in [G-M-P 2] and $[\mathrm{G}-\mathrm{M}-\mathrm{P} 4]$. In the first part of this paper we prove the corresponding theorem in the semi-local situation by building on the methods developed in these papers. There are two main steps: First we show that if $K$ is algebraically closed then $\boldsymbol{V}$ is principal. Next, using this case we show that the assertion that the sets $\boldsymbol{V}$ are principal is equivalent to the semi-local Skolem property for the existence of $\mathcal{V}$-integral points on varieties defined over $K$ (see section 2), and that this in turn is equivalent to a geometric criterion for the existence of divisors having prescribed poles and zeros in given open sets. When the valuations in $\mathcal{V}$ have rational rank one and residue fields which are algebraic over finite fields we are able to show that this geometric criterion is satisfied by a generalisation of the Rumely existence theorem. Thus by the end of the semi-local part we have proved theorem 1 when any one of the conditions (i), (ii) or (iii) is satisfied.

Globally, this result is more difficult to prove, as now one has to work with infinitely many valuations on the base simultaneously when finding the function $t$ that makes $\boldsymbol{V}$ principal. For geometric families of constant reductions we know that for almost all $v \in \mathcal{V}$ there is a unique prolongation to $F$ in $\boldsymbol{V}$ which is principal and has potential good reduction (see section 3 ). For the finitely many remaining elements of $\mathcal{V}$ one shows that the subset of prolongations in $\boldsymbol{V}$ is principal by the first part. Using Roquette's unit density lemma, [R2], we show that by suitably approximating the functions making the sets principal, after a finite base extension one obtains a global function which does the job. Finally, by taking the norm one obtains the global result for $\boldsymbol{V}$.

The theorem above can be used to give the following birational characterization of arithmetic surfaces, and prove the existence of finite morphisms to $\mathbb{P}^{1}$. This is the second theorem we want to emphasize here.

Let $S$ be a normal, integral, affine scheme whose local rings at the closed points are valuation rings, and suppose $\mathcal{V}$ is the corresponding set of valuations. Let $\mathcal{X}$ be a proper, normal, integral $S$-curve (=S-scheme of pure relative dimension 1$)$, 
with $K:=\kappa(S)$ relatively algebraically closed in $F:=\kappa(\mathcal{X})$. For each closed point $\mathcal{M}_{v} \in S$ let $\mathcal{O}_{\eta_{i}, v} \subseteq F, 1 \leq i \leq n_{v}$, be the local rings corresponding to the generic points of the irreducible components of the closed fibre at $\mathcal{M}_{v}$. We call the set of points of $\mathcal{X}$ corresponding to these local rings the embedded generic set at $v$. For each $i, \mathcal{O}_{\eta_{i}, v}$ is a valuation ring dominating $\mathcal{O}_{v}$ whose residue field is a function field of one variable over the residue field of $\mathcal{O}_{v}$, i.e., a constant reduction of $F$. Let $\boldsymbol{V}$ denote the family of constant reductions of $F$ corresponding to the embedded generic sets at each $v \in \mathcal{V}$. Using the above terminology we prove:

Theorem 2. Let $S$ and $\mathcal{X}$ be as defined above and suppose the family $\mathcal{V}$ satisfies one of the conditions in theorem 1. Then there is a finite morphism $\mathcal{X} \longrightarrow \mathbb{P}_{S}^{1}$. Moreover, up to isomorphism $\mathcal{X}$ is uniquely determined by $F=\kappa(\mathcal{X})$ and the family $\boldsymbol{V}$, which is geometric. Conversely, up to isomorphism each geometric family of constant reductions $\boldsymbol{V}$ of $F$ prolonging $\mathcal{V}$ determines a unique proper, normal, integral $S$ curve $\mathcal{X}$.

I would like to thank Michel Matignon, Florian Pop and Peter Roquette for many discussions related to these problems. The observation that in the global context, by using the norm one obtains a finite morphism to $\mathbb{P}_{S}^{1}$ without making a base change, was made by Florian Pop and also by Laurent Moret-Bailly in a private communication. I am grateful to them for suggesting this improvement.

\section{THE SEMI-LOCAL SITUATION}

Algebraically closed case. Let $F \mid K$ be given with $K$ algebraically closed, and suppose $\mathcal{V}$ and $\boldsymbol{V}$ are finite sets of independent valuations of $K$, respectively constant reductions prolonging $\mathcal{V}$ to $F$. Our aim is to show that $\boldsymbol{V}$ is principal, i.e., there exists $f \in F$ such that $\boldsymbol{V}=\boldsymbol{V}_{f}$. The non-constant function $f$ is called an element with the uniqueness property for $\boldsymbol{V}$.

2.1. Suppose $\mathcal{V}=\left\{v_{1}, \ldots, v_{n}\right\}$ and $\boldsymbol{V}=\bigcup_{i} V_{i}$, where $V_{i}=\left\{\mathrm{v} \in \boldsymbol{V}|\mathrm{v}|_{K}=v_{i}\right\}$. For each $i$, we let $\mathbf{v}_{i}(x)=\inf \mathrm{v}(x)$, for $\mathrm{v} \in V_{i}$ and $x \in F$. We set $F \mathbf{v}_{i}:=\mathcal{O}_{\mathbf{v}_{i}} / \mathcal{M}_{\mathbf{v}_{i}}$, where $\mathcal{O}_{\mathbf{v}_{i}}=\bigcap_{\mathrm{v} \in V_{i}} \mathcal{O}_{\mathrm{v}}$ and $\mathcal{M}_{\mathbf{v}_{i}}=\bigcap_{\mathrm{v} \in V_{i}} \mathcal{M}_{\mathrm{v}}$, the intersections being taken over the respective valuation rings and valuation ideals. Then $F \mathbf{v}_{i}:=\mathcal{O}_{\mathbf{v}_{i}} / \mathcal{M}_{\mathbf{v}_{i}} \cong$ $\prod_{\mathrm{v} \in V_{i}} \mathcal{O}_{\mathrm{v}} / \mathcal{M}_{\mathrm{v}}=\prod_{\mathrm{v} \in V_{i}} F \mathrm{v}$, the product of the residue fields of $F$ with respect to the constant reductions $\mathrm{v}$ via the identification $x+\mathcal{M}_{\mathbf{v}_{i}} \mapsto\left(x+\mathcal{M}_{\mathrm{v}}\right)_{\mathrm{v}}$. For $x \in F$ with $\mathbf{v}_{i}(x) \geq 0$ we let $x \mathbf{v}_{i}$ denote its image in $F \mathbf{v}_{i}$, and for a subset $M \subseteq F$ we denote the image of its elements of inf-norm $\geq 0$ by $M \mathbf{v}_{i}$.

For each $i$, let $\mathcal{X}_{V_{i}}$ be the proper, normal, integral $\mathcal{O}_{v_{i}}$-curve associated with $V_{i}$ as defined in [G-M-P 3], section 1 . Let $X$ be its generic fibre and $\mathcal{X}_{V_{i} \mathcal{M}_{i}}$ the special fibre at the closed point $\mathcal{M}_{i} \in \operatorname{Spec} \mathcal{O}_{v_{i}}$. By $[\mathrm{G}-\mathrm{M}-\mathrm{P} 2]$, proposition 2.5, and [G-M-P 3], lemma 1.3, the following assertions hold:

i) There exist finite subsets $A_{i} \subset \mathcal{X}_{V_{i} \mathcal{M}_{i}}$ with the property that if $D$ is a positive divisor of $F \mid K$ such that the specialisations of $\operatorname{supp}(D)$ lie in the complement of $A_{i}$ and meet all components of the closed fibre $\mathcal{X}_{V_{i} \mathcal{M}_{i}}$, then for large $n \in \mathbb{N}$ there exist $f_{i} \in F$ with $\left(f_{i}\right)_{\infty}=n D$ and $V_{f_{i}}=V_{i}$. 
ii) Contraction. For $U_{i} \subset V_{i}$ there is a commutative diagram of $\mathcal{O}_{v_{i}}$-curves:

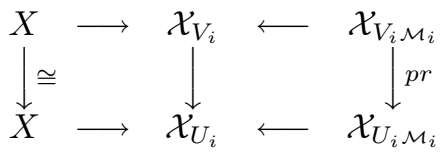

where the columns are surjective. Moreover there exist finite subsets $B_{i} \subset \mathcal{X}_{U_{i} \mathcal{M}_{i}}$ which have the following properties:

- $B_{i}$ has the property i).

- The preimage $T_{i}=\mathrm{pr}^{-1}\left(B_{i}\right)$ contains finite subsets $A_{i} \subset \mathcal{X}_{V_{i} \mathcal{M}_{i}}$ having the property i) and satisfying $\operatorname{pr}\left(A_{i}\right)=B_{i}$.

- $T_{i}$ contains a finite subset which can be prescribed in advance.

Lemma 2.2. For each $i, 1 \leq i \leq n$, let $U_{i} \subset V_{i}$ and $A_{i} \subset T_{i}$ be as in ii) above. Let $D$ be a divisor of $F \mid K$ and suppose that for each $i$, the specialisation of $\operatorname{supp}(D)$ in $\mathcal{X}_{V_{i} \mathcal{M}_{i}}$

- lies in the complement of the $A_{i}$;

- meets all the components determined by the $U_{i}$;

- has no contribution in the components determined by $V_{i} \backslash U_{i}$.

Then there exists $f \in F$ which is simultaneously a $U_{i}$ element with the uniqueness property for each $i, 1 \leq i \leq n$.

Proof. By the observations above, for each $i$ there exists a $U_{i}$ element with the uniqueness property $f_{i}$ with $\left(f_{i}\right)_{\infty}=m D$, for $m$ large enough. We define $f$ as follows: For each $i$ we apply the strong approximation theorem to obtain $a_{i} \in K$ with $v_{i}\left(a_{i}-1\right)>0$ and $v_{j}\left(a_{i}\right) \gg 0, j \neq i$. Setting $f=\sum_{i=1}^{n} a_{i} f_{i}$, it follows that $\operatorname{deg} f \leq m \operatorname{deg} D$, while for each $i$

$$
f \mathbf{v}_{i}=\sum_{j}\left(a_{j} f_{j}\right) \mathbf{v}_{i}=f_{i} \mathbf{v}_{i}, \quad \text { where } \quad \mathbf{v}_{i}=\inf _{\mathbf{v} \in U_{i}} \mathbf{v} .
$$

Now $\operatorname{deg} f_{i} \mathbf{v}_{i}=\operatorname{deg} f_{i}=m \operatorname{deg} D$, so it follows that $\operatorname{deg} f=\operatorname{deg} f_{i} \mathbf{v}_{i}=\operatorname{deg} f \mathbf{v}_{i}$. Therefore $f$ is a $U_{i}$ element with the uniqueness property for each $i$.

Lemma 2.3. Suppose that $\#\left(U_{i}\right)=\#\left(V_{i}\right)=1$ for each $i$ with $1 \leq i<n$, and suppose that $f \in F$ is a $V_{i}$ element with the uniqueness property for such $i$. Let $V_{n}$ be the set of all prolongations of the Gauß valuation $v_{n, f}$ from $K(f)$ to $F$, and fix $U_{n} \subset V_{n}$ with $\#\left(U_{n}\right)=1$. Then there exists a prime divisor $P$ of $F$ such that for $i$, $1 \leq i \leq n$, the specialisation is disjoint from $A_{i}$, and for $i=n$ it has contribution in the component corresponding to $U_{n}$.

Proof. For each $i$ choose $b_{i} \in K v_{i}$ so that $\left(\operatorname{supp}\left(f \mathbf{v}_{i}-b_{i}\right)_{0}\right) \cap A_{i}=\phi$. Next, using the chinese remainder theorem, choose $a \in K$ with $a v_{i}=b_{i}$ for all $i$.

We consider $\operatorname{supp}(f-a)_{0}$ : For each $i$ the specialisation is $\operatorname{supp}\left(f \mathbf{v}_{i}-b_{i}\right)_{0}$, so disjoint from $T_{i}$. For $i=n$ some $P \in \operatorname{supp}(f-a)_{0}$ has specialisation with contribution in the component of $\mathcal{X}_{V_{n} \mathcal{M}_{n}}$ determined by $U_{n}$.

Theorem 2.4. Let $F \mid K, \mathcal{V}$ and $\boldsymbol{V}$ be as described in 2.1. Then $\boldsymbol{V}$ is principal at $\mathcal{V}$.

Proof. We have $\mathcal{V}=\left\{v_{1}, \ldots, v_{n}\right\}$ and $\boldsymbol{V}=\bigcup_{i=1}^{n} V_{i}$. Set $r=\max _{i} \#\left(V_{i}\right)$. The theorem is proved by induction on $r$.

$r=1$ : To prove this case we now perform a second induction on $n=\#(\mathcal{V})$. 
For $n=1$ : This is the content of [G-M-P 2], theorem 3.1.

The step $n \rightarrow n+1$ : Suppose $f \in F$ is a $V_{i}$ element with the uniqueness property for $1 \leq i \leq n$, where $V_{i}=\left\{\mathrm{v}_{i}\right\}$. Let $\mathrm{v}_{n+1}$ be a constant reduction of $F$ prolonging $v_{n+1}$, and choose a polynomial $g=p(f) \in K[f]$ such that $g$ is a $V_{i}$ element with the uniqueness property for $1 \leq i \leq n$ and is $\mathrm{v}_{n+1}$ residually transcendental. Set $U_{i}=V_{i}, 1 \leq i \leq n, U_{n+1}=\left\{\mathrm{v}_{n+1}\right\}$, and let $V_{n+1}$ be the set of prolongations of the Gauß valuation $v_{n+1, g}$ on $K(g)$ to $F$. Next we apply lemma 2.3 to find a prime divisor $P$ of $F$ with specialisation disjoint from $A_{i}, 1 \leq i \leq n+1$, and for $i=n+1$ with specialisation in the component corresponding to $\mathrm{v}_{n+1}$. Now applying lemma 2.2 we obtain a function, say $h$, which is an element with the uniqueness property for $\mathrm{v}_{i}, 1 \leq i \leq n+1$.

To complete the proof of the theorem it remains to prove the inductive step $r \rightarrow r+1$. We partition the sets $V_{i}, 1 \leq i \leq n$, as follows:

i) if $\#\left(V_{i}\right)=1$ define $W_{i}=W_{i}^{\prime}=V_{i}$;

ii) if \# $\left(V_{i}\right)>1$ let $W_{i} \cup W_{i}^{\prime}=V_{i}$ be a proper partition.

Then for each $i=1, \ldots, n$, \# $\left(W_{i}\right), \#\left(W_{i}^{\prime}\right) \leq r$. Therefore by the inductive hypothesis there exist elements with the uniqueness property, $f$ for $W_{1}, \ldots, W_{n}$ and $g$ for $W_{1}^{\prime}, \ldots, W_{n}^{\prime}$. We next make adjustments to $f$ and $g$ as follows. Replace $f$ by $f_{1}$ and $g$ by $g_{1}$, elements with the uniqueness property for the respective sets $W_{1}, \ldots, W_{n}$ and $W_{1}^{\prime}, \ldots, W_{n}^{\prime}$, such that

a) $\mathbf{w}_{i}^{\prime}\left(f_{1}\right)>0$ and $\mathbf{w}_{i}\left(g_{1}\right)>0$ if $i$ is in case ii)

(here $\mathbf{w}_{i}^{\prime}=\inf _{\mathrm{v} \in W_{i}^{\prime}} \mathrm{v}$, resp. $\mathbf{w}_{i}=\inf _{\mathrm{v} \in W_{i}} \mathrm{v}$ ).

b) $f_{1} \mathrm{v}_{i}$ and $g_{1} \mathrm{v}_{i}$ have disjoint pole divisors if $i$ is in case i).

Now set $h=f_{1}+g_{1}$; then by degree considerations $h$ is an element with the uniqueness property for $V_{1}, \ldots, V_{n}$.

We explain how $f_{1}$ and $g_{1}$ are obtained from $f$ and $g$. Repeatedly we will have to use the chinese remainder theorem for the independent valuations $v_{1}, \ldots, v_{n}$ of $K$ to choose suitable coefficients when building $f_{1}$ and $g_{1}$ as rational functions of $f$ and $g$.

First we adjust $f$ and $g$ so that for each $i$ with $\#\left(V_{i}\right)=1, f \mathrm{v}_{i}$ and $g \mathrm{v}_{i}$ have no common zeros or poles. The second step is to construct $f_{1}$ which is a $W_{i}$ element with the uniqueness property, with $\mathrm{v}\left(f_{1}\right)>0$ for $\mathrm{v} \in V_{i} \backslash W_{i}, i$ as in case ii): Consider all $\mathrm{v} \in V_{i} \backslash W_{i}$ such that $\mathrm{v}(f)=0$. Then as $\mathrm{v} \notin W_{i}, f$ isn't $\mathrm{v}$ residually transcendental and we can choose $a_{\mathrm{v}} \in K$ with $f \mathrm{v}=a_{\mathrm{v}} \mathrm{v}$ and $v_{k}\left(a_{\mathrm{v}}\right)=0, k \neq i$. We have $\mathrm{v}\left(f-a_{\mathrm{v}}\right)>0$. Now taking the $f-a_{\mathrm{v}}$ for each $i$ as in case ii) and $\mathrm{v} \in V_{i} \backslash W_{i}$ as above, we form the product

$$
u=f \prod_{i} \prod_{\mathrm{v}}\left(f-a_{\mathrm{v}}\right) .
$$

Observe that $u$ is a $W_{i}$ element with the uniqueness property for $1 \leq i \leq n$. Let $f_{1}=u /(1+u)^{2} ;$ then for $\mathrm{v} \in V_{i} \backslash W_{i}$ :

- if $\mathrm{v}(f)=0$ then $\mathrm{v}(u)>0$ and so $\mathrm{v}\left(f_{1}\right)>0$;

- if $\mathrm{v}(f)>0$ then $\mathrm{v}(u)>0$ and so $\mathrm{v}\left(f_{1}\right)>0$

- if $\mathrm{v}(f)<0$ then $\mathrm{v}(u)<0$ and so $\mathrm{v}\left(f_{1}\right)=-\mathrm{v}(u)>0$

Note that $\left(f_{1}\right)_{\infty}=2(1+u)_{0}$.

Next we choose $g_{1}$, a $W_{i}^{\prime}$ element with the uniqueness property with $\mathrm{v}\left(g_{1}\right)>0$, for $\mathrm{v} \in V_{i} \backslash W_{i}^{\prime}, i$ as in case ii), in the same way as above but satisfying the additional 
requirement that after constructing

$$
t=g \prod_{i} \prod_{\mathrm{v}}\left(g-b_{\mathrm{v}}\right)
$$

we set $g_{1}=t /(c+t)^{2}$, with $c \in K$ chosen (using the chinese remainder theorem) so that for $i$ as in case i) the support of $\left(c v_{i}+t \mathrm{v}_{i}\right)_{0}$ is disjoint from that of $\left(1+u \mathrm{v}_{i}\right)_{0}$, and for $i$ as in case ii) $c v_{i}=1$. It then follows that $f_{1}$ and $g_{1}$ satisfy both a) and b), finishing the proof.

The semi-local Skolem property. Our aim in this subsection is to show that theorem 2.4 remains true when we descend from an algebraically closed field to one satisfying the semi-local Skolem property. When considering a global field $K$ equipped with a non-archimedean valuation, the local Skolem property is deduced from the work of Rumely [Ru1], more precisely from his Jacobian Principle which ensures the existence of functions having zeros in a given open and prescribed $K$ rational poles. In [G-M-P 4] we have shown that this strong geometric criterion is satisfied whenever the valuation on $K$ has rational rank 1 and the residue field is algebraic over a finite field. Thus in this situation the local Skolem property holds. Here we shall show that, using this strong geometric criterion, it follows that if $K$ is a field equipped with finitely many distinct valuations all having rational rank 1 and residue fields algebraic over finite fields, then the semi-local Skolem property is satisfied.

The results proved in this subsection generalise those of $[\mathrm{G}-\mathrm{M}-\mathrm{P} 4]$ and are needed to treat the semi-local situation.

First we need to recall the definitions.

Let $K$ be any field equipped with finitely many independent valuations $\mathcal{V}=$ $\left\{v_{1}, \ldots, v_{n}\right\}$, and $\tilde{K}$ be an algebraic closure equipped with fixed prolongations $\tilde{\mathcal{V}}=$ $\left\{\tilde{v}_{1}, \ldots, \tilde{v}_{n}\right\}$. For each $i$ let $\left(K_{i}^{\mathrm{h}}, v_{i}^{\mathrm{h}}\right)$ be the henselisation of $\left(K, v_{i}\right)$ which prolongs to $\left(\tilde{K}, \tilde{v}_{i}\right)$. We set $\mathcal{O}_{\mathcal{V}}=\bigcap_{i} \mathcal{O}_{v_{i}}$.

Let $X$ be a geometrically integral curve defined over $K$ with function field $F$, and denote by $X(\tilde{K})$ its set of $\tilde{K}$-rational points. For a tuple $\boldsymbol{x}=\left(x_{1}, \ldots, x_{m}\right)$, $x_{j} \in F$, and $S \subset \tilde{K}$ we define the $S$-rational points in $X(\tilde{K})$ with respect to $x$ by $X_{\boldsymbol{x}}(S):=\left\{P \in X(\tilde{K}): x_{j}(P) \in S, 1 \leq j \leq m\right\}$. The curve $X$ is said to satisfy the semi-local Skolem property at $\mathcal{V}$, if for each tuple $\boldsymbol{x}=\left(x_{1}, \ldots, x_{m}\right), x_{j} \in F$,

$$
X_{\boldsymbol{x}}\left(\mathcal{O}_{\tilde{v}_{i}}\right) \neq \varnothing \text { for each } i \Rightarrow X_{\boldsymbol{x}}\left(\tilde{\mathcal{O}}_{\mathcal{V}}\right) \neq \varnothing
$$

Density. If $X$ satisfies the semi-local Skolem property, then for each $i,\left(K, v_{i}\right)$ is dense in $\left(K_{i}^{\mathrm{h}}, v_{i}^{\mathrm{h}}\right)$. See $[\mathrm{G}-\mathrm{M}-\mathrm{P} 4]$, the proof of lemma 1.1.

Reciprocity and observations concerning the sets $X_{\boldsymbol{x}}\left(\mathcal{O}_{\tilde{v}_{i}}\right)$. Let $i$ be fixed, $\tilde{W}_{i}$ a set of constant reductions of $F \tilde{K}$ prolonging $\tilde{v}_{i}$ and suppose $x \in F \tilde{K}$ is a $\tilde{W}_{i}$ element with the uniqueness property. To simplify notation we supress the index $i$. Let $\mathcal{X}_{\tilde{W}}$ be the associated $\mathcal{O}_{\tilde{v}}$-curve as in 2.1 , with special fibre $\mathcal{X}_{\tilde{W} \tilde{\mathcal{M}}}:=\bar{X}$, and let $\bar{X}^{\prime}$ be its normalisation. The ring $\mathcal{O}_{\tilde{v}}[x]^{\prime}$ determines an affine open of $\mathcal{X}_{\tilde{W}}$, and $\mathcal{O}_{\tilde{v}}[x]^{\prime} \mathbf{v} \cong \mathcal{O}_{\tilde{v}}[x]^{\prime} \otimes_{\tilde{K}} \tilde{K} \tilde{v}$ the corresponding open dense subset of $\bar{X}$. Here $\tilde{\mathbf{v}}=\inf \tilde{\mathbf{v}}$ $(\tilde{\mathrm{v}} \in \tilde{W})$, the inf-norm on $F \tilde{K}$ determined by $\tilde{W}$, and the ring of rational functions of $\bar{X}$ is $F \tilde{K} \tilde{\mathbf{v}}=\prod F \tilde{K} \tilde{\mathbf{v}}$. The integral closure of $\mathcal{O}_{\tilde{v}}[x]^{\prime} \tilde{\mathbf{v}}$ in $F \tilde{K} \tilde{\mathbf{v}}$ is $\prod \tilde{K} \tilde{v}[x \tilde{\mathbf{v}}]^{\prime}$ and determines an affine open of $\bar{X}^{\prime}$. 
Given $P \in X(\tilde{K})$, let $\bar{P}$ be its specialisation in $\bar{X}$ and $\bar{Q}$ any point of $\bar{X}^{\prime}$ lying over $\operatorname{supp}(\bar{P})$. Then if $t \in \mathcal{O}_{\tilde{v}}[x]^{\prime}$ and $P \in X_{x}\left(\mathcal{O}_{\tilde{v}}\right)$, it follows that

$$
t(P) \tilde{v}=t \tilde{\mathrm{v}}(\bar{Q})
$$

where $\bar{Q}$ lies in the component of $\bar{X}^{\prime}$ determined by $\tilde{\mathrm{v}}$.

Indeed, as $t \in \mathcal{O}_{\tilde{v}}[x]^{\prime}$ and $P \in X_{x}\left(\mathcal{O}_{\tilde{v}}\right)$, it follows that $\tilde{v}(t(P)) \geq 0$. Using square brackets to denote the ideals of the points in the respective affine rings, we have

$$
(t-t(P)) \subset[P] \subset \mathcal{O}_{\tilde{v}}[x]^{\prime}, \quad(t-t(P)) \tilde{\mathbf{v}} \subset[\bar{P}] \subset \mathcal{O}_{\tilde{v}}[x]^{\prime} \tilde{\mathbf{v}}
$$

and hence $(t-t(P)) \tilde{\mathrm{v}} \subset[\bar{Q}] \subset \tilde{K} \tilde{v}[x \tilde{\mathrm{v}}]^{\prime}$. It follows that

$$
t \tilde{\mathrm{v}}(\bar{Q})-t(P) \tilde{v}=(t-t(P)) \tilde{\mathrm{v}}(\bar{Q})=0 .
$$

Using the observations above we see that for $P \in X_{x}\left(\mathcal{O}_{\tilde{v}}\right)$ and $\bar{Q}$ and $\tilde{\mathrm{v}}$ as above, if $t(P)$ is a $\tilde{v}$-unit then

- $\bar{Q} \notin \operatorname{supp}(t \tilde{\mathrm{v}})$.

- $t$ is a $\tilde{\mathrm{v}}$-unit.

Set $\boldsymbol{x}=\left(x, t, t^{-1}\right)$; then the scheme theoretical specialisation of $X_{\boldsymbol{x}}\left(\mathcal{O}_{\tilde{v}}\right)$ in $\bar{X}$ is disjoint from $\operatorname{supp}(t \tilde{\mathbf{v}})$ and the components determined by those $\tilde{\mathbf{v}} \in \tilde{W}$ for which $t$ isn't a $\tilde{\mathrm{v}}$-unit.

Prolongations of constant reductions. For a finite set $V_{i}$ of constant reductions of $F \mid K$ prolonging $v_{i}$ let $V_{i}^{\mathrm{h}}$ be the set of all constant reductions $\mathrm{v}_{i}^{\mathrm{h}}$ of $F K_{i}^{\mathrm{h}} \mid K_{i}^{\mathrm{h}}$ which prolong both $V_{i}$ and $v_{i}^{\mathrm{h}}$ to $F K_{i}^{\mathrm{h}}$. We define $\tilde{V}_{i}$ correspondingly on $F \tilde{K}$, and remark that $\left.\tilde{V}_{i}\right|_{F K_{i}^{\mathrm{h}}}=V_{i}^{\mathrm{h}}$ and $\left.V_{i}^{\mathrm{h}}\right|_{F}=V_{i}$. Further by [G-M-P 4], for each $\mathrm{v}_{i} \in V_{i}$ there is a unique $\mathrm{v}_{i}^{\mathrm{h}} \in V_{i}^{\mathrm{h}}$ prolonging it.

Theorem 2.5. Let $X$ be a geometrically integral curve defined over $K$ with function field $F$, and $\mathcal{V}$ be a finite set of independent valuations of $K$. Then the following are equivalent.

i) $X$ has the semi-local Skolem property at $\mathcal{V}$.

ii) Each finite set of constant reductions $\boldsymbol{V}$ of $F$ prolonging $\mathcal{V}$ is principal.

iii) Geometric criterion: For each $i,\left(K, v_{i}\right)$ is dense in its henselisation $\left(K_{i}^{\mathrm{h}}, v_{i}^{\mathrm{h}}\right)$, and for every $\boldsymbol{x}=\left(x_{1}, \ldots, x_{m}\right), x_{j} \in F$, if $X_{\boldsymbol{x}}\left(\mathcal{O}_{\tilde{v}_{i}}\right) \neq \varnothing$ for all $i$, then there exists a positive $K$-rational divisor $D$ and, for each $i, P_{i, 1}, \ldots, P_{i, d} \in X_{\boldsymbol{x}}\left(\mathcal{O}_{\tilde{v}_{i}}\right)$ such that $D \sim P_{i, 1}+P_{i, 2}+\cdots+P_{i, d}$ over $\tilde{K}$.

Proof. i) $\Rightarrow$ ii) First let $x \in F$ be chosen residually transcendental for each $\mathrm{v} \in \boldsymbol{V}$. For each $i, 1 \leq i \leq n$, we let $W_{i}$ be the set of prolongations of $v_{i, x}$ to $F$ and set $\boldsymbol{W}=\bigcup_{i} W_{i}$. Observe that as $V_{i} \subset W_{i}$ for each $i, \boldsymbol{V} \subset \boldsymbol{W}$. Let $\tilde{\boldsymbol{V}}=\bigcup_{i} \tilde{V}_{i}$, where $\tilde{V}_{i}$ is the set of constant reductions of $F \tilde{K}$ prolonging both $V_{i}$ and $\tilde{v}_{i}$; we define $\tilde{\boldsymbol{W}}=\bigcup_{i} \tilde{W}_{i}$ similarly.

For each $i$, let $A_{i}$ be a finite subset of $\mathcal{X}_{\tilde{W}_{i} \tilde{\mathcal{M}}_{i}}$. Then as $x$ is $W_{i}$ residually transcendental, there exists a polynomial $z \in \mathcal{O}_{\mathcal{V}}[x]$ such that $A_{i}$ is contained in the specialisation to $\mathcal{X}_{\tilde{W}_{i} \tilde{\mathcal{M}}_{i}}$ of the divisor support of $z$, for each $i$. We choose the sets $A_{i}$ to satisfy 1.1 i) for the closed fibre $\mathcal{X}_{\tilde{W}_{i} \tilde{\mathcal{M}}_{i}}$. Next we choose $y \in \mathcal{O}_{\mathcal{V}}[x]^{\prime} \subset F$ such that for each $i, y \mathrm{v} \neq 0$ for $\mathrm{v} \in V_{i}$ and $y \mathrm{v}=0$ for $\mathrm{v} \in W_{i} \backslash V_{i}$.

Let $\boldsymbol{x}=\left(x, y, y^{-1}, z, z^{-1}\right)$ and for each $i$ let $\mathcal{D}_{i}$ be the scheme theoretical specialisation of $X_{\boldsymbol{x}}\left(\mathcal{O}_{\tilde{v}_{i}}\right)$ in $\mathcal{X}_{\tilde{W}_{i} \tilde{\mathcal{M}}_{i}}$. Then, by the discussion preceeding the theorem, for each $i, \mathcal{D}_{i}$ is disjoint from the set $A_{i}$ and the components of $\mathcal{X}_{\tilde{W}_{i} \tilde{\mathcal{M}}_{i}}$ corresponding to $\tilde{\mathrm{v}} \in \tilde{W}_{i} \backslash \tilde{V}_{i}$. It follows that $\mathcal{X}_{\tilde{W}_{i} \tilde{\mathcal{M}}_{i}} \backslash \mathcal{D}_{i}$ has the properties required of $T_{i}$ in 1.1 ii) (here $W_{i}$ replaces $V_{i}$ and $V_{i}$ replaces $U_{i}$ ). In particular, for every $K$-rational 
divisor $D$ with $\operatorname{supp}(D) \subset X_{\boldsymbol{x}}\left(\mathcal{O}_{\tilde{v}_{i}}\right)$ whose specialisation meets all the components of $\mathcal{X}_{\tilde{V}_{i} \tilde{\mathcal{M}}_{i}}$, there exist $\tilde{V}_{i}$ elements with the uniqueness property $h_{i} \in F \tilde{K}$ such that $\left(h_{i}\right)_{\infty}=l D$ for $l \in \mathbb{N}$ big enough.

Since $X_{\boldsymbol{x}}\left(\mathcal{O}_{\tilde{v}_{i}}\right) \neq \varnothing$ for each $i$, by hypothesis there exists $P \in X_{\boldsymbol{x}}\left(\tilde{\mathcal{O}}_{\mathcal{V}}\right)$. We let $D=r \sum P^{\prime}$, where $P^{\prime}$ is a system of $G_{K}$ conjugates of $P$ and $r$ is a large enough power of the characteristic exponent of $K$ so that $D$ is $K$-rational. By the discussion above we conclude that for each $i$ there exists a $\tilde{V}_{i}$ element with the uniqueness property $h_{i} \in F \tilde{K}$ with $\left(h_{i}\right)_{\infty}=l D$, provided $l$ is large enough.

Directly from the definition it follows that $\tilde{V}_{i}$ is $G_{K_{i}^{\mathrm{h}}}$ invariant. Hence for each $\sigma \in G_{K_{i}^{\mathrm{h}}}$ one has $\tilde{V}_{i}^{\sigma}=\tilde{V}_{i}$, and consequently $\sigma h_{i}$ is a $\tilde{V}_{i}$ element with uniqueness property with $\left(h_{i}\right)_{\infty}=\left(\sigma h_{i}\right)_{\infty}$. Set $t_{i}=\left(\prod \bar{\sigma} h_{i}\right)^{r}$, where the $\bar{\sigma} h_{i}$ are a system of representatives for the $G_{K_{i}^{\mathrm{h}}}$ conjugates of $h_{i}$ and $r$ is a large enough power of the characteristic exponent of $K$ so that $t_{i} \in F K_{i}^{\mathrm{h}}$. As the $\bar{\sigma} h_{i}$ all have the same pole divisor, it follows that $t_{i}$ is a $\tilde{V}_{i}$ element with the uniqueness property. Since it lies in $F K_{i}^{\mathrm{h}}$, it is an element with the uniqueness property for $V_{i}^{\mathrm{h}}$. We denote the pole divisor of $t_{i}$ by $m D$.

Now as $\left(K, v_{i}\right)$ is dense in $\left(K_{i}^{\mathrm{h}}, v_{i}^{\mathrm{h}}\right)$ we can find $g_{i}$ in the linear space $\mathcal{L}_{K}(m D)$ such that $\left(g_{i}\right)_{\infty}=m D$ and $\mathrm{v}_{i}^{\mathrm{h}}\left(t_{i}-g_{i}\right)>0$ for $\mathrm{v}_{i}^{\mathrm{h}} \in V_{i}^{\mathrm{h}}$. Then, using the fundamental inequality,

$$
\begin{aligned}
{\left[F K_{i}^{\mathrm{h}}: K_{i}^{\mathrm{h}}\left(t_{i}\right)\right] } & =\left[F K_{i}^{\mathrm{h}}: K_{i}^{\mathrm{h}}\left(g_{i}\right)\right] \\
& \geq \sum e_{\mathrm{v}_{i}^{\mathrm{h}}} \delta_{\mathrm{v}_{i}^{\mathrm{h}}}\left[F K_{i}^{\mathrm{h}} \mathrm{v}_{i}^{\mathrm{h}}: K_{i}^{\mathrm{h}}\left(g_{i}\right) \mathrm{v}_{i}^{\mathrm{h}}\right] \quad \text { as } \mathrm{v}_{i}^{\mathrm{h}} \in V_{i g_{i}}^{\mathrm{h}} \\
& \geq \sum e_{\mathrm{v}_{i}^{\mathrm{h}}} \delta_{\mathrm{v}_{i}^{\mathrm{h}}}\left[F K_{i}^{\mathrm{h}} \mathrm{v}_{i}^{\mathrm{h}}: K_{i}^{\mathrm{h}}\left(t_{i}\right) \mathrm{v}_{i}^{\mathrm{h}}\right]=\left[F K_{i}^{\mathrm{h}}: K_{i}^{\mathrm{h}}\left(t_{i}\right)\right]
\end{aligned}
$$

Thus $\left[F K_{i}^{\mathrm{h}}: K_{i}^{\mathrm{h}}\left(g_{i}\right)\right]=\sum e_{\mathrm{v}_{i}^{\mathrm{h}}} \delta_{\mathrm{v}_{i}^{\mathrm{h}}}\left[F K_{i}^{\mathrm{h}} \mathrm{v}_{i}^{\mathrm{h}}: K_{i}^{\mathrm{h}}\left(g_{i}\right) \mathrm{v}_{i}^{\mathrm{h}}\right]$, and hence $g_{i}$ is an element with the uniqueness property for $V_{i}^{\mathrm{h}}$. Since $g_{i} \in F$, it is an element with uniqueness property for $V_{i}$.

Setting $g=\sum_{i} a_{i} g_{i}, a_{i} \in K$ chosen as in 1.2 so that $g \mathbf{v}_{i}=g_{i} \mathbf{v}_{i}$, it follows that for each $i, g$ is a $V_{i}$ element with the uniqueness property, completing the proof of i) $\Rightarrow$ ii).

ii) $\Rightarrow$ i) Suppose $\boldsymbol{x}=\left(x_{1}, \ldots, x_{m}\right), x_{j} \in F$, and for each $i, 1 \leq i \leq n, X_{\boldsymbol{x}}\left(\mathcal{O}_{\tilde{v}_{i}}\right) \neq \varnothing$. We show that $X_{\boldsymbol{x}}\left(\tilde{\mathcal{O}}_{\mathcal{V}}\right) \neq \varnothing$. We first show that for each $i$ there exists $f_{i} \in F K_{i}^{\mathrm{h}}$ such that $X_{f_{i}}\left(\mathcal{O}_{\tilde{v}_{i}}\right) \subset X_{\boldsymbol{x}}\left(\mathcal{O}_{\tilde{v}_{i}}\right)$.

Note that for $P \in X_{\boldsymbol{x}}\left(\mathcal{O}_{\tilde{v}_{i}}\right)$ all its $\mathrm{G}_{K_{i}^{\mathrm{h}}}$-conjugates $P^{\prime}$ lie in $X_{\boldsymbol{x}}\left(\mathcal{O}_{\tilde{v}_{i}}\right)$. Set $A=$ $p^{e} \sum P^{\prime}$, where $p$ is the characteristic exponent of $K$ and $e$ is sufficiently large so that $A$ is $K_{i}^{\mathrm{h}}$-rational and by Riemann-Roch there exists $g_{i} \in F K_{i}^{\mathrm{h}}$ with $\left(g_{i}\right)_{0}=A$. Next let $u_{j}(T):=\operatorname{Irr}\left(x_{j}(P) \mid K_{i}^{\mathrm{h}}\right)$ and set $y_{j}=u_{j}\left(x_{j}\right)$. Clearly $\operatorname{supp} A \subset \operatorname{supp}\left(y_{j}\right)_{0}$; hence $g_{i}^{-1}$ is integral over $\tilde{K}\left[y_{j}^{-1}\right]$. From the irreducible equations of $g_{i}^{-1}$ over the $\tilde{K}\left[y_{j}^{-1}\right]$, one deduces that for all $\pi_{i} \in K_{i}^{\mathrm{h}}$ with $v_{i}^{\mathrm{h}}\left(\pi_{i}\right)$ sufficiently large, $f_{i}=g_{i} / \pi_{i}$ satisfies the claim.

Let $V_{f_{i}}^{\mathrm{h}}$ be the set of all prolongations of the Gauß valuation $v_{i f_{i}}^{\mathrm{h}}$ to $F K_{i}^{\mathrm{h}}$, and $V_{i}$ its restriction to $F$. By the earlier remarks we have $V_{f_{i}}^{\mathrm{h}}=V_{i}^{\mathrm{h}}$.

Let $g \in F$ be an element with the uniqueness property for $V_{i}$ for each $i$, which exists by hypothesis. Then by the earlier remarks it follows that $g$ is an element with the uniqueness property for $V_{i}^{\mathrm{h}}$, for each $i$. It follows that $f_{i}$ is integral over 
$\mathcal{O}_{v_{i g}^{\mathrm{h}}}$ and so also over $\mathcal{O}_{\tilde{v}_{i g}}$ when viewed in $F \tilde{K} \mid \tilde{K}$. Hence one has

$$
f_{i}^{n_{i}}+r_{i, n_{i}-1}(g) f_{i}^{n_{i}-1}+\cdots+r_{i, 0}(g)=0,
$$

where $r_{i, k}(g)=p_{i, k}(g) / q_{i, k}(g)$ and $\tilde{v}_{i g}\left(q_{i k}(g)\right)=0 \leq \tilde{v}_{i g}\left(p_{i, k}(g)\right)$. By the maximum principle there exists $\alpha_{i} \in \mathcal{O}_{\tilde{v}_{i}}$ such that $\tilde{v}_{i}\left(q_{i, k}\left(\alpha_{i}\right)\right)=0 \leq \tilde{v}_{i}\left(p_{i, k}\left(\alpha_{i}\right)\right)$. Using the strong approximation theorem we choose $\alpha \in \tilde{K}$ approximating the $\alpha_{i}$ closely enough with respect to $\tilde{v}_{i}$ so that

$$
\tilde{v}_{i}\left(q_{i, k}(\alpha)\right)=0 \leq \tilde{v}_{i}\left(p_{i, k}(\alpha)\right) .
$$

We show that the zeros $P$ of $g-\alpha$ lie in $X_{f_{i}}\left(\mathcal{O}_{\tilde{v}_{i}}\right)$ : Let $P$ be a zero of $g-\alpha$, i.e. $g(P)=\alpha$. Then

$$
\left(f_{i}(P)\right)^{n_{i}}+r_{i, n_{i}-1}(\alpha)\left(f_{i}(P)\right)^{n_{i}-1}+\cdots+r_{i, 0}(\alpha)=0 .
$$

As the $r_{i, k}(\alpha) \in \mathcal{O}_{\tilde{v}_{i}}$ it follows that $f_{i}(P) \in \mathcal{O}_{\tilde{v}_{i}}$. Hence all the zeros $P$ of $h=g-\alpha$ lie in $X_{f_{i}}\left(\mathcal{O}_{\tilde{v}_{i}}\right) \subset X_{\boldsymbol{x}}\left(\mathcal{O}_{\tilde{v}_{i}}\right)$.

Next we set $h_{i}=\left(\prod_{\bar{\sigma}} \bar{\sigma} h\right)^{r}$, where the $\bar{\sigma} h$ are a system of representatives for the $G_{K_{i}^{\mathrm{h}}}$-conjugates of $h$ and $r$ is a suitable power of the characteristic exponent such that $h_{i} \in F K_{i}^{\mathrm{h}}$. Note that for each $i$ we have $\operatorname{supp}\left(h_{i}\right)_{0} \subset X_{\boldsymbol{x}}\left(\mathcal{O}_{\tilde{v}_{i}}\right)$. Let $L \mid K$ be a finite galois extension over which each of the $h_{i}$ are defined, and set $v_{i L}=\left.\tilde{v}_{i}\right|_{L}$. For each $i$ the set of all prolongations of $v_{i}$ to $L$ can be described by $\left(v_{i L}^{\bar{\tau}_{i}}\right)_{\bar{\tau}_{i}}$, where the $\bar{\tau}_{i}$ form a system of representatives for the cosets of $\operatorname{Gal}(L \mid K) / Z\left(v_{i L} \mid v_{i}\right)$. For each $\bar{\tau}_{i}$ let $\bar{\tau}_{i} h_{i}$ be the corresponding conjugate of $h_{i}$. As $K$ is dense in $K_{i}^{\mathrm{h}}$ it follows that the valuations $\left(v_{i L}^{\bar{\tau}_{i}}\right)_{\bar{\tau}_{i}, i}$ are independent. Therefore the diagonal embedding $L \hookrightarrow \prod_{i} \prod_{\bar{\tau}}\left(L, v_{i L}^{\bar{\tau}_{i}}\right)$ is dense, and so is the embedding $\mathcal{L}_{L}(D) \hookrightarrow \prod_{i} \prod_{\bar{\tau}_{i}}\left(\mathcal{L}_{L}(D), v_{i L}^{\bar{\tau}_{i}}\right)$. Hence, there exist $t \in \mathcal{L}_{L}(D)$ such that $t \approx \bar{\tau}_{i} h_{i}$ in the $v_{i L}^{\bar{\tau}_{i}}$-adic topology for each $\bar{\tau}_{i}$. Given $\sigma \in G_{K}$, for each $i$ let $\bar{\tau}_{i}$ be its representative in $\operatorname{Gal}(L \mid K) / Z\left(v_{i L} \mid v_{i}\right)$. Then $\sigma h_{i}=\bar{\tau}_{i} h_{i}$ and hence $\sigma^{-1} t \approx h_{i}$ in the $\tilde{v}_{i}$-adic topology, as $t \approx \bar{\tau}_{i} h_{i}$ in the $v_{i L}^{\bar{\tau}_{i}}$-adic topology. Consequently for each $i$ the zeros of $\sigma^{-1} t$ are $\tilde{v}_{i}$-close to those of $h_{i}$. By the choice of $h_{i}, \operatorname{supp}\left(h_{i}\right)_{0} \subseteq X_{\boldsymbol{x}}\left(\mathcal{O}_{\tilde{v}_{i}}\right)$; hence the same is true for the zeros of $\sigma^{-1} t$. Therefore, if $P$ is a zero of $t$ then $P^{\sigma} \in X_{\boldsymbol{x}}\left(\mathcal{O}_{\tilde{v}_{i}}\right)$ for all $\sigma \in G_{K}$ and $i$. Equivalently, $P \in X_{\boldsymbol{x}}\left(\mathcal{O}_{\tilde{v}_{i}^{\sigma}}\right)$ for all $\sigma \in G_{K}$ and $i$. Hence $P \in X_{\boldsymbol{x}}\left(\tilde{\mathcal{O}}_{\mathcal{V}}\right)$, completing the proof of ii) $\Rightarrow$ i).

Equivalence with iii). The interested reader can easily modify the proof of ii) $\Rightarrow$ i), which essentially goes through iii). The only additional information needed for the direction iii) $\Rightarrow \mathrm{i}$ ), is that if the $P_{i, j} \in X_{\boldsymbol{x}}\left(\mathcal{O}_{\tilde{v}_{i}}\right)$ are not disjoint from supp $D$ then one can replace them by points for which this is the case; see [G-M-P 4] for details.

For our discussion we shall not use the geometric criterion above, but a stronger form which is valid when the valuations $v_{i}$ have rational rank 1 and residue fields which are algebraic over finite fields. In this situation we have:

2.6. The strong geometric criterion. Let $X$ be a geometrically integral curve defined over $K$ with function field $F$, and $\mathcal{V}=\left\{v_{1}, \ldots, v_{n}\right\}$ a finite set of distinct valuations of $K$ having rational rank 1 and residue fields algebraic over finite fields. Then for every $\boldsymbol{x}=\left(x_{1}, \ldots, x_{m}\right), x_{j} \in F$, if $X_{\boldsymbol{x}}\left(\mathcal{O}_{\tilde{v}_{i}}\right) \neq \varnothing$ for all $i$, then for each positive $K$-rational divisor $D$ there exist $P_{i, 1}, \ldots, P_{i, d} \in X_{\boldsymbol{x}}\left(\mathcal{O}_{\tilde{v}_{i}}\right)$ such that, for some multiple, $m D \sim P_{i, 1}+P_{i, 2}+\cdots+P_{i, d}$ over $\tilde{K}$. 
This result is part of the proof of [G-M-P 4], theorem 3.1, and is established using the non-archimedean uniformisation of abelian varieties when $\mathcal{V}$ consists of only 1 valuation, and so holds generally. Using this strong geometric criterion, we obtain:

Theorem 2.7. Let $X$ be a geometrically integral curve defined over $K$ with function field $F$ and $\mathcal{V}$ a finite set of distinct valuations of $K$ having rational rank 1 and residue fields algebraic over finite fields. Then $X$ has the semi-local Skolem property at $\mathcal{V}$.

Proof. By the discussion above, $X$ satisfies the strong geometric criterion at $\mathcal{V}$. Therefore, given $\boldsymbol{x}=\left(x_{1}, \ldots, x_{m}\right), x_{j} \in F$, if $X_{\boldsymbol{x}}\left(\mathcal{O}_{\tilde{v}_{i}}\right) \neq \varnothing$ for all $i$, then for each positive $K$-rational divisor $D$ there exist $f_{i} \in F \tilde{K}$ with $\left(f_{i}\right)_{\infty}=m D$ for some multiple and $\operatorname{supp}\left(f_{i}\right)_{0} \subset X_{\boldsymbol{x}}\left(\mathcal{O}_{\tilde{v}_{i}}\right)$. Using the $f_{i}$ we construct functions $h_{i}$ defined over $F K_{i}^{\mathrm{h}}$ as in the last part of $\left.2.5 \mathrm{ii}\right) \Rightarrow \mathrm{i}$ ), and so deduce there exist $P \in$ $X_{\boldsymbol{x}}\left(\tilde{\mathcal{O}}_{\mathcal{V}}\right)$

Remarks. 1) As a corollary to theorem 2.7 we obtain theorem 1, stated at the beginning of the paper, subject to the conditions i), ii) or iii) (the semi-local part).

2) For an arbitrary field $K$ equipped with finitely many independent valuations $\mathcal{V}$ the semi-local Skolem property implies the local Skolem property for each $v \in \mathcal{V}$. When the $v$ have rational rank 1 and residue fields algebraic over finite fields, the converse also holds. However, it is an open question whether this equivalence holds in general.

\section{The PASsage From the SEMI-LOCAL TO THE GLOBAL Situation}

Our aim in this section is to extend the results obtained in the semi-local situation to the global situation. The main ingredients used here are Roquette's Unit Density Property and principal basis construction with regularity criterion for constant reductions.

We begin by fixing the notations to be used throughout this section.

3.1. 1) Let $K$ be an arbitrary field endowed with a set $\mathcal{V}$ of non-archimedean valuations of finite character. This means that for each $a \in K, v(a)=0$ for almost all $v \in \mathcal{V}$. Further we assume that the set of valuations $\mathcal{V}$ satisfies the strong approximation property. Note that if $K^{\prime}$ is any finite extension of $K$, then the prolongation $\mathcal{V}^{\prime}$ of $\mathcal{V}$ has the same properties. See for example [Grif].

2) Next we recall those results from constant reduction theory which are fundamental to our discussion. For details the reader is referred to $[\mathrm{G}-\mathrm{M}-\mathrm{P} 1],[\mathrm{P} 1]$, [P2], [R1], and [R3].

Let $F \mid K$ be a function field in one variable and $t \in F$ a non-constant function. Then by [G-M-P 1], 1.3, and [R1], there exists a finite set $A \subset K$ such that for each non-archimedean valuation $v$ of $K$, if $v(A) \geq 0$ then the Gauß valuation $v_{t}$ has a unique prolongation $\mathrm{v}$ to $F$ and $t$ is regular at v, i.e. $\operatorname{deg} t=\operatorname{deg} t \mathrm{v}$. Further there exists a finite purely inseparable constant extension $F K^{\prime} \mid K^{\prime}$ of $F \mid K$ such that the unique prolongation $\mathrm{v}^{\prime}$ of $\mathrm{v}$ to $F K^{\prime}$ is a good reduction. We say that $\mathrm{v}$ is a potential good reduction of $F \mid K$.

The families of constant reductions we are interested in satisfy the following geometric property. 
Definition. Geometric families of constant reductions. Let $\boldsymbol{V}$ be a set of constant reductions of $F$ prolonging $\mathcal{V}$. Then $\boldsymbol{V}$ is said to be geometric if there exists a nonconstant function $t \in F$ such that $\boldsymbol{V}$ and $\boldsymbol{V}_{t}:=\bigcup_{v \in \mathcal{V}} V_{t}$ are almost equal. Here by almost equal we mean that the symmetric difference of $\boldsymbol{V}$ and $\boldsymbol{V}_{t}$ is finite.

Observations. 1) The set $\boldsymbol{V} \mid \mathcal{V}$ determined by the $S$-curve $\mathcal{X}$ from the introduction (theorem 2) is a geometric set of constant reductions. (See the proof of theorem 2 at the end of the paper).

2) By the remarks in 3.12 ), if $t$ and $u$ are non-constant functions of $F$, then $\boldsymbol{V}_{t}$ and $\boldsymbol{V}_{u}$ are almost equal. Hence it follows that any two geometric sets of constant reductions prolonging $\mathcal{V}$ are almost equal.

3) Using 2) above together with 3.12 ), we deduce that $\boldsymbol{V}$ is a geometric set of constant reductions if and only if its prolongation to any finite extension $F K^{\prime} \mid K^{\prime}$ is.

Therefore without loss of generality we can assume that for almost all $v \in \mathcal{V}$ there is a unique prolongation $\mathrm{v}$ to $F$ in $\boldsymbol{V}$ and that $\mathrm{v}$ is a potential good reduction. Let $\mathcal{G} \subset \mathcal{V}$ be the set of all such valuations.

3.2. The Unit Density Property. The pair $(K, \mathcal{V})$ is said to satisfy the Unit Density Property if for each finite subset $\mathcal{S} \subset \mathcal{V}$ and $a \in \tilde{K}^{\times}$there exists $c \in \tilde{K}$, such that:

- $c-a$ is arbitrarily close to $0 v$-adically for all $v \in \mathcal{S}$. (In general $c, a \notin K$, and to say that $c-a$ is arbitrarily close to $0 v$-adically means that this is the case for every prolongation of $v$ to $\tilde{K}$.)

- $c$ is a unit at $v$ for all $v \in \mathcal{V} \backslash \mathcal{S}$. (Again, this means that $c$ is a unit at each prolongation of $v$ to $\tilde{K}$.)

The unit density property is satisfied whenever $\mathcal{O}_{\mathcal{V}}$ is a Dedekind domain whose ideal class group is torsion and the valuations have residue fields which are algebraic over a finite field. Furthermore, the algebraic number $c$ can be chosen so that each prime from $\mathcal{S}$ splits completely in $K(c)$, i.e., $K(c)$ is contained in $K^{\mathcal{S}}$, the field of totally $\mathcal{S}$-adic numbers. In particular, if $K$ is a global field and $\mathcal{V}$ is any set of non-archimedean valuations satisfying the strong approximation property, then the unit density property is satisfied. This is a non-trivial theorem, which together with the Rumely existence theorem are the main ingredients needed to prove the Local-Global Principle for the solvability of algebraic diophantine equations in the ring of all algebraic integers over a global field. A proof of the unit density theorem can be found in $[\mathrm{C}-\mathrm{R}]$, and the application to prove the Local-Global Principle in $[\mathrm{G}-\mathrm{P}-\mathrm{R}]$.

Throughout the rest of this section we shall assume that $K$ is equipped with a set of non-archimedean valuations having the unit density property.

3.3. The principal basis and regularity criterion. Suppose $D$ is a positive divisor of $F \mid K$, and for each prime divisor $Q \in \operatorname{supp}(D)$ let $r_{Q}$ denote its multiplicity and $l_{Q}:=\operatorname{deg} Q$. From the Riemann-Roch theorem it follows that for each $Q \in$ $\operatorname{supp}(D)$ and $m>2 g_{F}$ there exist functions $u_{m Q i}, 1 \leq i \leq l_{Q}$,

- with pole divisor $m Q$ and zeros disjoint from the primes occuring in $D$, and

- which are $K$-linearly independent and hence form a $K$-basis for $\mathcal{L}(m Q) \bmod$ ulo $\mathcal{L}((m-1) Q)$.

Now let $n>2 g_{F}$, and for each $Q$ set $n_{Q}=n r_{Q}$. Then, again applying RiemannRoch, the functions $\left(u_{n_{Q} Q i}\right)_{Q, i}$ form a $K$-basis of $\mathcal{L}(n D)$ modulo $\mathcal{L}(B)$, where 
$B=\sum_{Q}\left(n_{Q}-1\right) Q$. We will call this basis a principal basis for $D$ of level $n$, and to simplify the notation we set $u_{Q i}=u_{n_{Q} Q i}$. We next choose a $K$-basis of $\mathcal{L}(B)$, say $\left(w_{j}\right)_{j}$. Then each function $f \in \mathcal{L}(n D)$ has a unique representation in the form

$$
f=\sum_{j} c_{j} w_{j}+\sum_{Q, i} c_{Q i} u_{Q i}
$$

with coefficients $c_{j}, c_{Q i} \in K$. The pole divisor of $f$ is precisely $n D$ if and only if $c_{Q i} \neq 0$ for all $Q$ and some $i$.

Regularity criterion. Let $v \in \mathcal{V}$, and $\mathrm{v}$ a constant reduction of $F \mid K$ prolonging $v$. For the basis $\left(w_{j}\right)_{j} \cup\left(u_{Q i}\right)_{Q, i}$ of $\mathcal{L}(n D)$ we assume that:

i) The $w_{j}$ and the $u_{Q i}$ are regular functions for $\mathrm{v}$.

ii) For each $Q$, the functions $u_{Q i}, 1 \leq i \leq l_{Q}$, form a $v$ valuation basis for $\mathcal{L}\left(n_{Q} Q\right)$ modulo $\mathcal{L}\left(\left(n_{Q}-1\right) Q\right)$, and for $Q \neq Q^{\prime}$ one has

$$
\operatorname{supp}\left(u_{Q i} \mathrm{v}\right)_{\infty} \cap \operatorname{supp}\left(u_{Q^{\prime} k} \mathrm{v}\right)_{\infty}=\varnothing \text {. }
$$

From these assumptions it follows that

- for each $Q$ and $i \neq k,\left(u_{Q i} \mathrm{v}\right)_{\infty}=\left(u_{Q k} \mathrm{v}\right)_{\infty}=n_{Q} \bar{Q}$, for some divisor $\bar{Q} \in$ $\operatorname{Div}(F \mathrm{v} \mid K v)$;

- for each function $w_{j}, \operatorname{supp}\left(w_{j} \mathrm{v}\right)_{\infty} \subset \bigcup_{Q} \operatorname{supp} \bar{Q}$;

- the functions $\left(u_{Q i}\right)_{Q, i}$ form a $v$ valuation basis for $\mathcal{L}(n D)$ modulo $\mathcal{L}(B)$.

Let $f \in F$ with $(f)_{\infty}=n D$, so that it can be written in the form

$$
f=\sum_{j} c_{j} w_{j}+\sum_{Q, i} c_{Q i} u_{Q i}, \quad c_{j}, c_{Q i} \in K .
$$

Suppose that $v\left(c_{j}\right) \geq 0$ for each $j$, and $v\left(c_{Q i}\right)=0$ for each $Q$ and $i$. Then $f$ is a regular function for v. Indeed $\left(w_{j} \mathrm{v}\right)_{\infty}<\sum_{\bar{Q}} n_{Q} \bar{Q}$, so for $f$ we obtain

$$
\begin{aligned}
\operatorname{deg} f \mathrm{v} & =\sum_{Q} \operatorname{deg} w_{Q} \mathrm{v}, \quad \text { where } w_{Q} \mathrm{v}:=\sum_{i} c_{Q i} u_{Q i} \mathrm{v}, \\
& =\sum_{Q} n_{Q} \operatorname{deg} \bar{Q}=\operatorname{deg} f .
\end{aligned}
$$

To simplify notation we shall simply write $\left(u_{i}\right)_{i}$ when we mean the basis $\left(w_{j}\right)_{j} \cup$ $\left(u_{Q i}\right)_{Q, i}$ of $\mathcal{L}(n D)$.

We now prove the main theorem of this section.

Theorem 3.4. Let $F \mid K$ be a function field in one variable and $\mathcal{V}$ a set of valuations of the constant field $K$ satisfying:

i) the semi-local Skolem property for $F \mid K$;

ii) the unit density property.

Let $\boldsymbol{V} \mid \mathcal{V}$ be a geometric set of constant reductions of $F$. Then $\boldsymbol{V}$ is principal at $\mathcal{V}$.

Proof. It suffices to prove the theorem when $F \mid K$ is a regular function field. For otherwise, let $E|K \subset F| K$ be a function field in one variable with $E$ separably closed in $F$. Then $E \mid K$ is regular and each constant reduction of $E$ has a unique prolongation to $F$. Hence there is a bijective correspondence between the constant reductions of $F$ and $E$, via restriction and prolongation. Therefore if a geometric set of constant reductions of $E$ is principal, so is its prolongation to $F$.

Suppose $\mathcal{S}_{1}=\mathcal{V} \backslash \mathcal{G}$, and let $\boldsymbol{V}_{\mathcal{S}_{1}}$ be the set of all prolongations of the valuations of $\mathcal{S}_{1}$ in $\boldsymbol{V}$. Then by definition both these sets are finite. Hence by the semi-local Skolem property there exists $f \in F$ which is a $\boldsymbol{V}_{\mathcal{S}_{1}}$ element with the uniqueness 
property. Suppose $(f)_{\infty}=n D$, with $n>2 g_{F}$. (We replace $f$ by some power if necessary.)

Next let $\left(u_{i}\right)_{i}$ be a principal basis for $n D$. Then, by assumption, for almost all $v \in \mathcal{G}$ the $u_{i}$ satisfy the conditions $3.3 \mathrm{i}$ ) and ii) for the potential good reduction v prolonging $v$. Suppose $\mathcal{S}_{2} \subset \mathcal{G}$ is the finite subset for which these conditions are not satisfied. Then after a finite purely inseparable extension of $F$, the potential good reduction $\mathrm{v}$ prolongs to a good reduction which we again denote by $\mathrm{v}$. Using the divisor reduction map for each such $\mathrm{v}$, see [R3], plus the chinese remainder theorem, we construct a function $h$, simultaneously v-regular for each $\mathrm{v} \mid v, v \in \mathcal{S}_{2}$, with $(h)_{\infty}=n D$.

Using the principal basis construction, we have

$$
f=\sum a_{i} u_{i} \quad \text { and } \quad h=\sum b_{i} u_{i}, \quad \text { with } \quad a_{i}, \in K, b_{i} \in \tilde{K} .
$$

We shall now use the unit density property to construct a function having the required properties.

Using the unit density property, we find $c_{i} \in \tilde{K}$ such that

- $c_{i}-a_{i}$ is arbitrarily close to $0 v$-adically for $v \in \mathcal{S}_{1}$;

- $c_{i}-b_{i}$ is arbitrarily close to $0 v$-adically for $v \in \mathcal{S}_{2}$

- $c_{i}$ is a unit at $v$ for all $v \in \mathcal{V} \backslash\left(\mathcal{S}_{1} \cup \mathcal{S}_{2}\right)$.

Setting $\mathcal{S}=\mathcal{S}_{1} \cup \mathcal{S}_{2}$ and $K^{\prime}=K\left[\left(c_{i}\right)_{i}\right]$, it follows that $K^{\prime} \subset K^{\mathcal{S}}$.

We set $t=\sum c_{i} u_{i} \in F K^{\prime}$ and claim it is a $\boldsymbol{V}^{\prime} \mid \mathcal{V}^{\prime}$ element with the uniqueness property. Here $\boldsymbol{V}^{\prime} \mid \mathcal{V}^{\prime}$ is the prolongation of $\boldsymbol{V} \mid \mathcal{V}$ to $F K^{\prime} \mid K^{\prime}$.

For $v^{\prime} \in \mathcal{S}_{1}^{\prime}$, by writing $t=f+(t-f)$, observing that for each $\mathrm{v}^{\prime} \in V^{\prime}$, $t \mathrm{v}^{\prime}=f \mathrm{v}^{\prime}+(t-f) \mathrm{v}^{\prime}=f \mathrm{v}^{\prime}$ while $\operatorname{deg} t=\operatorname{deg} f=n \operatorname{deg} D$, and applying the fundamental valuation equality (possibly with defect contribution), it follows that $t$ is a $V^{\prime}$ element with the uniqueness property.

For $v^{\prime} \in \mathcal{S}_{2}^{\prime}$ we write $t=h+(t-h)$ and note that it is $\mathrm{v}^{\prime}$-regular, as here $\operatorname{deg} t=\operatorname{deg} h=\operatorname{deg} h \mathrm{v}^{\prime}=\operatorname{deg} t \mathrm{v}^{\prime}$.

For $v^{\prime} \in \mathcal{V}^{\prime} \backslash\left(\mathcal{S}^{\prime}\right)$, we use the regularity criterion to ensure that $t$ is $\mathrm{v}^{\prime}$-regular.

In the last part of the proof we show that, considering the norm of $t$ in $F$, we obtain an element with the uniqueness property for $\boldsymbol{V} \mid \mathcal{V}$. For each $\sigma \in \operatorname{Aut}\left(F K^{\prime} \mid F\right)=$ $\operatorname{Aut}\left(K^{\prime} \mid K\right)$ (as $F \mid K$ is assumed to be regular) we have:

- $(\sigma t)_{\infty}=(t)_{\infty}$, as the pole divisor is $K$-rational.

- $\boldsymbol{V}^{\prime \sigma}=\boldsymbol{V}^{\prime}$ and $\mathcal{V}^{\prime \sigma}=\mathcal{V}^{\prime}$

We deduce that $\sigma t$ is an element with the uniqueness property for $\boldsymbol{V}^{\prime} \mid \mathcal{V}^{\prime}$. Finally, consider $h=\prod_{\sigma} \sigma t$. By the observations above, $h$ is also an element with the uniqueness property for $\boldsymbol{V}^{\prime} \mid \mathcal{V}^{\prime}$. Since $h \in F$, on restricting to $F$ we see that the same holds for $\boldsymbol{V} \mid \mathcal{V}$, completing the proof of the theorem.

Remarks. The remaining part of theorem 1, i.e., subject to the condition iv) (the global part), follows as a corollary to theorem 3.4.

Combining the above result with the characterisation for curves over valuation rings given in $[\mathrm{G}]$, we can now prove theorem 2 :

Proof of theorem 2. Recall, $S$ is a normal, integral, affine scheme whose local rings at the closed points are valuation rings, and $\mathcal{V}$ is the corresponding set of valuations, which is assumed to satisfy one of the conditions in theorem $1 . \mathcal{X}$ is a proper, 
normal, integral $S$-curve and $\boldsymbol{V}$ is the family of constant reductions of $F:=\kappa(\mathcal{X})$ prolonging $\mathcal{V}$ which are determined by its embedded generic set.

We first observe that by $[\mathrm{G}]$, theorem 3.6, it follows that up to a birational isomorphism $\mathcal{X}$ is uniquely determined by the family $\boldsymbol{V}$.

Next we note that because of the conditions imposed on $\mathcal{V}$, it follows that $\boldsymbol{V}$ is a geometric family of constant reductions. In the semi-local situation this is clear from the definitions. In the global situation $S=\operatorname{Spec} \mathcal{O}$, where $\mathcal{O}$ is some affine normal model for a global field $K$. For almost all $v \in \mathcal{V}$, the fibre over $\mathcal{M}_{v} \in S$ has potential good reduction. Therefore for such $v$ there is a unique prolongation $\mathrm{v}$ in $\boldsymbol{V}$, and this is a potential good reduction of $F$. By the observations made at the beginning of this section it follows that if $t$ is any non-constant function in $F$, the symmetric difference of $\boldsymbol{V}$ and $\boldsymbol{V}_{t}$ is finite. Hence $\boldsymbol{V}$ is a geometric family of constant reductions.

Applying theorem 1, we deduce that $\boldsymbol{V}$ is principal, with an element with the uniqueness property, $f$, say. We conclude that $\mathcal{X}$ is $S$-isomorphic to the normalisation of $\mathbb{P}_{S}^{1}$ relative to the extension of function fields, $F \mid K(f)$.

Finally note that on the other hand, if $\boldsymbol{V}$ is any geometric family of constant reductions of $F$ prolonging $\mathcal{V}$, one obtains the unique proper, normal, integral $S$ model $\mathcal{X}$ having $\boldsymbol{V}$ as embedded generic set by taking the normalisation of $\mathbb{P}_{S}^{1}$ relative to the extension $F \mid K(f)$. This completes the proof of theorem 2 .

\section{REFERENCES}

[C-R] D. Cantor, P. Roquette, On diophantine equations over the ring of all algebraic integers, J. Number Theory 18 (1984), 1-26. MR 85i:11036

[G] B. Green, On curves over valuation rings and morphisms to $\mathbb{P}^{1}$, J. Number Theory 59 (1996), 262-290. MR 97g:14024

[G-M-P 1] B. Green, M. Matignon, F. Pop, On valued function fields I, Manuscripta Math. 65 (1989), 257-276. MR 91g:12010

[G-M-P 2] _ On valued function fields II, Regular functions and elements with the uniqueness property, J. reine angew. Math. 412 (1990), 128-149. MR 92e:12008

[G-M-P 3] _ On valued function fields III, Reductions of algebraic curves, J. reine angew. Math. 432 (1992), 117-133. MR 94f:12004

[G-M-P 4] _ On the Local Skolem Property, J. reine angew. Math. 458 (1995), 183-199. MR 95k: 12012

[G-P-R] B. Green, F. Pop, P. Roquette, On Rumely's Local-Global Principle, Jber. d. Dt. Math.-Verein 97 (1995), 43-74. MR 96g:11065

[Grif] M. Griffin, Rings of Krull type, J. reine angew. Math. 229 (1968), 1-27. MR 36:3778

[M-O 1] M. Matignon, J. Ohm, A structure theorem for simple transcendental extensions of valued fields, Proc. Amer. Math. Soc. 104 (1988), 392-402. MR 90h:12011

[M-O 2] Simple transcendental extensions of valued fields III: The uniqueness property, J. Math. Kyoto Univ. 30-2 (1990), 347-365. MR 91i:12017

[M-B] L. Moret-Bailly, Groupes de Picard et problèmes de Skolem I, II, Ann. Scient. Éc. Norm. Sup. 22 (1989), 161-179, 181-194. MR 90i:11065

[P1] F. Pop, On the Galois Theory of function fields of one variable over number fields, J. reine angew. Math. 406 (1990), 200-218. MR 92d:11127

[P2] On Grothendieck's conjecture of birational anabelian geometry, Ann. of Math. 138 (1994), 145-182. MR 94m:12007

[R1] P. Roquette, Zur Theorie der Konstantenreduktion algebraischer Mannigfaltigkeiten, J. reine angew. Math. 200 (1958), 1-44. MR 20:4560

[R2] Solving diophantine equations over the ring of all algebraic integers, Atas de $8^{\mathrm{e}}$ Escola de Algebra, Vol. 2, IMPA 84.

[R3] $\quad$ Reciprocity in valued function fields, J. reine angew. Math. 375/376 (1987), 238-258. MR 88f:11058 
[Ru1] R. S. Rumely, Arithmetic over the ring of all algebraic integers, J. reine angew. Math. 368 (1986), 127-133. MR 87i:11041

[Ru2] Capacity Theory on Algebraic Curves, Springer-Verlag, Lecture Notes in Mathematics 1378, 1989. MR 91b:14018

Department of Mathematics, University of Stellenbosch, Stellenbosch 7602, South AFricA

E-mail address: bwg@land.sun.ac.za 